\title{
COVID-19 vaccination in autoimmune disease (COVAD) survey protocol
}

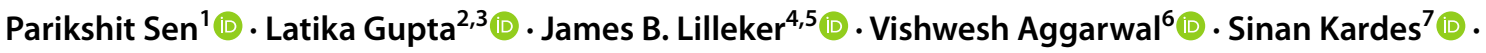

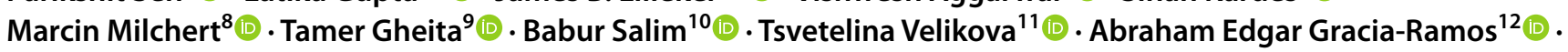 \\ loannis Parodis ${ }^{13,14}$. Albert Selva O'Callaghan ${ }^{15}$ (1) Elena Nikiphorou ${ }^{16,17}$ (1) Ai Lyn $\operatorname{Tan}^{18,19}$ (1)

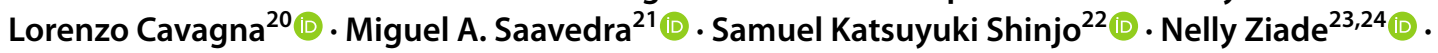

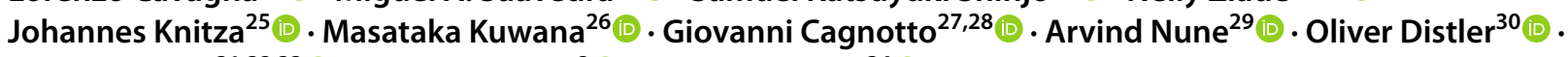

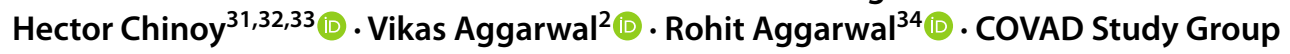

Received: 24 September 2021 / Accepted: 28 October 2021 / Published online: 15 November 2021

(c) Crown 2021

\begin{abstract}
The coronavirus disease-2019 (COVID-19) pandemic continues to be a cause of unprecedented global morbidity and mortality. Whilst COVID-19 vaccination has emerged as the only tangible solution to reducing poor clinical outcomes, vaccine hesitancy continues to be an obstacle to achieving high levels of vaccine uptake. This represents particular risk to patients with autoimmune diseases, a group already at increased risk of hospitalization and poor clinical outcomes related to COVID19 infection. Whilst there is a paucity of long-term safety and efficacy data of COVID-19 vaccination in patients with autoimmune diseases, the current evidence strongly suggests that the benefits of vaccination outweigh the risks of adverse effects and disease flares. Herein, we report the protocol of the COVID-19 Vaccination in Autoimmune Diseases (COVAD) study, an ongoing international collaborative study involving 29 countries and over 110 investigators.
\end{abstract}

Keywords COVID-19 $\cdot$ Autoimmune diseases $\cdot$ COVAD $\cdot$ Vaccination $\cdot$ Survey

\section{Introduction}

The ongoing coronavirus disease-2019 (COVID-19) global pandemic caused by the severe acute respiratory syndrome coronavirus 2 (SARS-CoV-2) continues to cause unprecedented morbidity and mortality, with even the most robust healthcare systems struggling to deliver consistent highquality care to patients with COVID-19, and the effects on other functions of the healthcare system. The only tangible way to reduce the socioeconomic burden and strain on healthcare systems due to COVID-19 is to achieve herd immunity against SARS-CoV-2 [1, 2]. In this respect, vaccination provides a ray of hope as a powerful tool for reducing the adverse outcomes of COVID-19. Current evidence suggests that patients with systemic autoimmune rheumatic diseases (AIRD) are at an increased risk of severe COVID-19

Members of The COVAD Study Group are listed in the Acknowledgrment section.

Latika Gupta

drlatikagupta@gmail.com

Rohit Aggarwal

aggarwalr@upmc.edu clinical outcomes, emphasizing the importance of COVID19 vaccination in this patient group [3]. However, a high degree of vaccine hesitancy continues to be a hurdle against vaccination in patients with autoimmune disease as well as in healthy individuals.

The exclusion of patients with AIRD from COVID-19 vaccine trials inevitably resulted in lacunae in the evidence about the long-term safety and efficacy of COVID-19 vaccination. There were also concerns about the efficacy of using COVID-19 vaccines in patients on immunosuppressive drugs and whether or not to stop medication. This unsurprisingly gave rise to fears of disease flares and adverse effects associated with vaccination in an already vulnerable group, amongst patients and healthcare professionals, increasing vaccine hesitancy $[4,5]$. The unprecedented disruption to the healthcare delivery system, most prominently the closure of many outpatient clinics due to their conversion to COVID-19 wards and redeployment of the staff, as well as the marked reduction in elective and preventive hospital visits by patients due to the imposition of travel restrictions, coupled with the advent of mainstream telemedicine services, had an inescapable effect on medical research [6]. The pandemic also had a positive effect of bringing together 
clinicians and researchers from around the world, united by the common goal of improving patient care, catalysing an increase in global networking for research [7].

To address the gaps in the current evidence, a global study group of doctors and researchers conceptualized the COVID-19 Vaccination in Autoimmune Diseases (COVAD) study, with the vision of collecting meaningful data on the post-vaccination adverse effects and their determinants in patients with AIRD, particularly patients with rare conditions such as idiopathic inflammatory myopathies, systemic sclerosis and other systemic autoimmune diseases assessing these effects in comparison to those observed in people without AIRD. The COVAD study is a long-term ongoing global patient self-reported survey study, with the goal of evaluating the long-term efficacy, as well as the short and long-term adverse effects and disease flares in AIRD patients vaccinated against COVID.

\section{Methods}

We developed an extensive self-report e-survey to assess the safety of the COVID-19 vaccine in idiopathic inflammatory myopathies and other AIRDs, and non-autoimmune controls. The questionnaire was vetted by international experts, pilot tested, validated and translated into 18 languages, before being hosted on the surveymonkey.com platform. Back and forth translations were performed by two independent reviewers per language. The survey questions were designed to evaluate previous COVID-19 infection, current vaccination status, and short-term (within 1-2 weeks) as well as long term (1-12 months) adverse drug effects following vaccine administration.

\section{Literature review}

A search was conducted using PubMed, MEDLINE, EMBASE, and SCOPUS databases, using the combination of the keywords "COVID-19 OR SARS-CoV2 OR SARSCOV-2 OR novel coronavirus OR nCOV" AND "vaccination OR vaccine OR immunization OR immunization" AND "autoimmune disease OR AID OR rheumatic disease OR autoimmune inflammatory rheumatic disease OR AIRD" AND "side effect OR adverse effect OR adverse reaction". Additional searches were performed with the terms "signs OR symptoms OR effects OR features OR clinical features OR clinical characteristics". Approximately, 2300 articles were identified and ranged from case reports, case series, and letters to the editor, and observational studies to narrative reviews and systematic reviews. Relevant articles were independently reviewed and used to formulate the survey questionnaire. Initially, 34 questions were generated. The questions "Who confirmed your autoimmune disease diagnosis?" and "In which year were you were diagnosed with autoimmune disease?" were added, as well as questions regarding details of symptoms including "What kind of rashes did you notice?" and "If you have swelling in your joints, how many joints are swollen". Two questions regarding contact details including telephone number and email were excluded to preserve patient anonymity. Finally, there were 36 questions. Core item set included demographics, post-vaccination adverse effects symptom set as per standard CDC guidelines, and patient-reported outcome measures.

\section{Survey design}

The baseline questionnaire featured 36 Covid-19 and AIRDrelated questions, covering several areas including previous COVID-19 infection $(n=5)$, vaccination status $(n=5)$, short-term minor and major adverse effects $(n=2)$, diagnosis and current symptom status $(n=11)$, functional status $(n=3)$, treatment history $(n=6)$ and demographics $(n=4)$. Fifteen were multiple choice questions requiring a single answer option, while nine questions could have multiple answer options selected, three questions needed an answer to be selected from a drop-down list, two questions asked the respondents for a specific date or for a specific number of days, two questions asked the respondent to answer on a self-reporting scale, two were questions with five components each to be reported on a 5-point Likert scale, and two asked for contact details for follow-up. Choices closed ended in 24 questions with an "other (please specify)" option were deemed appropriate in 13 questions. The survey was designed by LG, reviewed by RA, and was translated into 18 languages. The survey is enclosed as Supplementary file 3. Data dictionary is provided as Supplementary file 4.

\section{Pilot testing and validation}

Four rheumatologists (RA, LG, VA and HC), one neurologist (JBL), and one undergraduate medical student (VA) reviewed the questions and confirmed them to be representative of the content and face validity. The questionnaire underwent extensive pilot testing with 50 rounds of dummy completions with 25 people to identify errors in wording grammar, and syntax, and critically evaluate face validity. 26 questions were either modified, added or deleted following pilot testing. The average survey time was 5 minutes. A logic function was applied in five questions to limit survey time. The respondents could change the answers before submission but not after it. All questions, except the two asking for contact details, were mandatory. 


\section{Population selection}

Participants were eligible to participate if they are were adults ( $>18$ years old), and either healthy, or diagnosed to have any autoimmune disease. Convenience sampling was used, and all those who agreed to participate were included in the survey. A brief explanation was provided in the cover letter obtained by clicking the survey link, which include details of the survey purpose, content, data handling, and investigator. It also sought informed consent for publication of results in a peer-reviewed journal.

The study was not specifically advertised as a study of vaccine hesitancy, thus minimizing bias by attracting people with strongly held views on the matter. No incentives were offered for survey completion.

\section{Consent and ethics approvals}

Informed consent was obtained at the beginning of the survey and no incentives were offered for survey completion. Approval of local institutional ethics committee was obtained as per local guidelines [8]. We adhered to the Checklist for Reporting Results of Internet e-surveys to report the data $[9,10]$ with electronic protocols to address checks were done to avoid duplicated responses from a single respondent.

\section{Survey dissemination}

An international group of 110 rheumatologists, internists, neurologists and immunologists from $>80$ healthcare centers in over 50 countries conducted the e-survey with their patients and their non-AIRD family members. In addition, the survey was floated on social media platforms (e.g. WhatsApp, Facebook, and Instagram) and among online patient support group members across the world. The survey was first launched in the first half of 2021 and shall be open until the 31st of December 2021. As of August 2021, 16,327 responses had accrued. We retrieved the hesitancy data from the baseline survey. Future surveys would be designed based on the same model and address concerns around long-term effects on disease and physical function, booster shots and breakthrough infections, and other issues arising after of COVID-19 vaccination.

\section{Statistical analysis}

Descriptive statistics would be used. Intergroup comparisons would be made as required by the research question. Openended responses in the "Others (please specify)" category would be manually allocated to existent categories, and a new category would be listed when the responses did not fit into an existent category. Data will be downloaded from surveymonkey.com into Microsoft Excel for analysis. Incomplete responses would be excluded at the time of analysis. Stata, R and SPSS would be used for future analysis.

\section{Future analyses from the dataset}

The anonymized dataset would be open to future analysis by the core team based on proposed hypothesis, research question and study design proposed by collaborators. A COVAD steering committee has been proposed to vet the proposals for scientific validity and feasibility. Relevant authorship guidelines have been formulated to this effect. A study request form has been devised to detail the research question, methods, data variables to be analysed, geographic region and time period of study, and proposed statistical analyses (Supplementary file 3).

\section{Dissemination of outcomes}

Results will be disseminated in peer-reviewed journals, via the media, online, and at academic conferences. A plain language summary of results from the study will be made available to participants upon request.

\section{Future research and data sharing}

Participants are invited to provide a unique identifier code with optional consent to be contacted for future research, such as further follow-up beyond 6 months. All information about the study (including design, project administration, and publication preprints) will be available on request [11].

\section{Project closure}

At the conclusion of the study, recruitment materials, the project landing page, and online survey materials will be deactivated or removed. All data will remain securely stored with PI for a period of 5 years.

\section{Discussion}

There is certainly an unmet need for further research into the long-term safety and efficacy of COVID-19 vaccination in patients with AIRD (24). The prospective design of the project is designed to provide timely information to federal and regulatory agencies regarding the emergent concerns around vaccination among individuals with autoimmune disease. This information will be useful to inform the development of relevant practices and screening tools to identify those at utmost risk of adverse events. It may also provide 
insight into the effect of current immunosuppressant use on the risk for adverse events, and potentially guide vaccine administration and even management post-vaccination based on immunosuppression level and disease-specific stratified risk sets. While the format of the study is a self-reporting survey, the addition of specific questions regarding verification of diagnosis of autoimmune disease by a healthcare professional"Who confirmed your autoimmune disease diagnosis?" as well as most respondents being patients with chronic AIRDs, who, with their familiarity of their condition and treatment, would be more likely to correctly report symptoms, adverse effects and treatment history, increases the validity of the data collected. The databank would provide further insights into risks incurrent by age, gender, ethnicity, and vaccine type, if any, which may be useful in refining more targetted vaccination approaches towards particular vulnerable groups and specific regions. Most importantly, this large ethnically and geographically diverse dataset will add to the valuable databank of information on the safety of COVID vaccines and curb misinformation [12, 13].

Furthermore, the findings of this research can guide policymakers on the various aspects of immunization that may be focussed on in future research to reduce imminent risks of immediate adverse events, short- and long-term side effects, and most importantly triggered autoimmunity in specific AIDs, and relevant groups such as pregnant and lactating women [14]. The COVID-19 pandemic has widened the communication gap between care providers and patients, and it is imperative to understand patient perspectives as gathered by this self-report survey. Finally, the findings from this study can be used to devise well-informed guidelines for families on how to deal with this unique situation to improve care and quality of life among people with AIDs whilst protecting them from imminent risks of contracting COVID-19.

Supplementary Information The online version contains supplementary material available at https://doi.org/10.1007/s00296-021-05046-4.

Acknowledgements The authors thank all respondents for filling the questionnaire. The authors thank The Myositis Association, Myositis India, Myositis UK, the Myositis Global Network, Cure JM, Cure IBM, Sjögren's India Foundation, EULAR PARE, and various other patient support groups and organizations for their invaluable contribution in the dissemination of this survey among patients which made the data collection possible. The authors also thank all members of the COVAD study group. List of collaborators of the COVAD Study Group. India: Dr. Bhupen Barman-Department of Medicine, North Eastern Indira Gandhi Regional Institute of Health and Medical Sciences (NEIGRIHMS), Shillong-18, Meghalaya. Dr. Yogesh Preet Singh, Assistant Professor, Division of Rheumatology and Clinical Immunology, Department of General Medicine, Himalayan Institute of Medical sciences, Swami Rama University, Jolly Grant, Dehradun-248,140, Uttarakhand, India. Dr. Rajiv Ranjan-Clinical Immunology \& Rheumatology at Columbia Asia, Palam Vihar, Gurgaon, Haryana. Dr. Avinash Jain, Assistant Professor, Department of Clinical Immunology and Rheumatology, SMS Medical College and Hospital, Jaipur, Rajasthan. Dr. Sapan C. Pandya, Director, Clinical Immunologist and
Rheumatologist, Rheumatic Disease Clinic, Vedanta Institute of Medical Sciences, Navrangpura, Ahmedabad 380,009, Gujarat. Dr. A. N. Malaviya, Senior Consultant, Indian Spinal Injuries Center and A\&R Clinic for Arthritis and Rheumatism, New Delhi, Delhi. Dr. Rakesh Kumar Pilania, MD, DM, Assistant Professor, Pediatric Allergy Immunology Unit, Department of Pediatrics, Post Graduate Institute of Medical Education and Research, Chandigarh. Dr. Aman Sharma, Professor, Clinical Immunology and Rheumatology Services, Department of Internal Medicine, Post Graduate Institute of Medical Education and Research, Chandigarh. Dr. Manesh Manoj M, Consultant, Department of Clinical Immunology and Rheumatology, AKG Memorial Hospital and Dr Shenoy's CARE (Centre for Arthritis and Rheumatism Excellence), Kannur, Kerala. Dr. Vikas Gupta, Assistant Professor (Rheumatology), Dayanand Medical College and Hospital, Ludhiana, Punjab 141,001, India. Dr. Chengappa G. Kavadichanda, Assistant Professor, Department of Clinical Immunology, Jawaharlal Institute of Postgraduate Medical Education and Research, Puducherry, India. Dr. Pradeepta Sekhar Patro, Consultant, Department of Clinical Immunology and Rheumatology, Sunshine Hospitals, Plot No 208, Cuttack Puri Road, Laxmisagar, Bhubaneshwar, Odisha. Dr. Sajal Ajmani, Consultant, Arthritis and Rheumatology clinic, New Delhi, Delhi. Dr. Sanat Phatak, Associate Consultant-Rheumatology \& Immunology, Department of Rheumatology and Immunology, KEM Hospital, Pune, Maharashtra. Dr. Rudra Prosad Goswami, Assistant Professor, Department of Rheumatology, All India Institute of Medical Sciences, New Delhi, Delhi. Dr. Abhra Chandra Chowdhury, Rheumatologist, AMRI Hospital, Dhakuria, Kolkata, West Bengal. Dr. Ashish Jacob Mathew, Dept Clinical Immunology \& Rheumatology, Christian Medical College and Hospital, Vellore, Tamil Nadu 632,004. Dr. Padnamabha Shenoy, Medical Director (CARE), Dr Shenoy's CARE (Centre for Arthritis and Rheumatism Excellence), Kannur, Kerala. Dr. Ajay Asranna, Department of Neurology, NIMHANS, Bengaluru, Karnataka. Dr. Keerthi Talari Bommakanti, Consultant Rheumatologist, Yashoda hospital, Behind Hari Hara Kala Bhavan, Secunderabad-500,003, T.S. Hyderabad, Telangana. Dr. Anuj Shukla, Niruj Rheumatology Clinic, 209 Rajvi Complex, Rambaug, Ahmedabad, 380,008, Gujarat. Dr. Naveen R, Department of Clinical Immunology and Rheumatology, Sanjay Gandhi Postgraduate Institute of Medical Sciences, Raebarelli Road-226014, Lucknow, Uttar Pradesh. Turkey: Dr. Döndü Üsküdar Cansu, Division of Rheumatology, Department of Internal Medicine, Eskişehir Osmangazi University, 26,480, Eskişehir, Turkey. United Kingdom: Dr. John D. Pauling, 1. Royal National Hospital for Rheumatic Diseases (at Royal United Hospitals), Upper Borough Walls, Bath, BA1 1RL, UK. 2. Department of Pharmacy and Pharmacology, University of Bath, Bath, UK. Dr. Chris Wincup, Department of Rheumatology, University College London, London, UK. United States: Dr. Tulika Chatterjee, Department of Internal Medicine, University of Illinois College of Medicine at Peoria, Illinois. Dr. Minchul Kim, PhDResearch Assistant Professor, Center for Outcomes Research, Department of Internal Medicine, University of Illinois College of Medicine Peoria, Illinois. France: Dr. Margherita Giannini, Explorations fonctionnelles musculaires, service de physiologie, Hôpitaux universitaires de Strasbourg; EA3072, fédération de médecine translationnelle. Italy: Dr. Nicoletta Del Papa, Unità operativa complessa (UOC) Day Hospital Reumatologia via Gaetano Pini 9, Centro Specialistico Ortopedico Traumatologico, Gaetano Pini-CTO, Milano, Italy. Dr. Gianluca Sambataro, Medico Immunologia e reumatologia presso, Artoreuma S.R.L., Cors S. Vito 53, 95,030 Mascalucia, CT, Italy. Dr. Atzeni Fabiola, Full Professor, Rheumatology Unit, University of Messina, Messina, Italy. Dr. Marcello Govoni, Professor, Department of Medical Sciences, Complex Operative Unit and Rheumatology Unit of S.Anna University Hospital, University of Ferrara, Via A. Moro 8, 44,124- Cona (FE), Italy. Dr. Simone Parisi, Epidemiology Unit, Italian Society for Rheumatology, Milan, Italy; Rheumatology Unit, Azienda Ospedaliera Città della Salute e della Scienza di Torino, Torino, Italy. Dr. Elena Bartoloni Bocci, Associate Professor, Department of Medicine and Surgery, 
MED/16- Rheumatology, Università degli studi di Perugia, P.zza Università-06,123 - Perugia, Italy. Dr. Gian Domenico Sebastiani, U.O.C. Reumatologia, Ospedale San Camillo-Forlanini, Roma, Italy. Dr. Enrico Fusaro, Rheumatology Unit, Azienda Ospedaliero-Universitaria Città della Salute e della Scienza di Torino, Torino, Italy. Dr. Marco Sebastiani, Rheumatology Unit, University of Modena and Reggio Emilia, Azienda Ospedaliero-Universitaria Policlinico di Modena, Via del Pozzo, 41,125, Modena, Italy. Dr. Luca Quartuccio, Clinic of Rheumatology, Department of Medicine (DAME), ASUFC, University of Udine, Udine, Italy. Dr. Franco Franceschini, Rheumatology and Clinical Immunology Unit, Department of Clinical and Experimental Sciences, ASST Spedali Civili and University of Brescia, Italy. Dr. Pier Paolo Sainaghi, Department of Translational Medicine, Università del Piemonte Orientale UPO, Novara, Italy; Division of Internal Medicine, Immunorheumatology Unit, CAAD (Center for Translational Research on Autoimmune and Allergic Disease) Maggiore della Carità Hospital, Novara, Italy; IRCAD, Interdisciplinary Research Center of Autoimmune Diseases, Novara. Dr. Giovanni Orsolini, Department of Medicine, Rheumatology Unit, University of Verona, Verona, Italy. Dr. Rossella De Angelis, Rossella De Angelis, Rheumatology Unit, Department of Clinical and Molecular Sciences, Polytechnic University of Marche. Dr. Maria Giovanna Danielli, Clinica Medica, Dipartimento di Scienze Cliniche e Molecolari, Università Politecnica delle Marche e Azienda Ospedali Riuniti, Ancona, Italy. Philippines: Dr. Lisa S. Traboco, Philippine Rheumatology Association, St Luke's Medical Center- Global City (Visiting), Philippines. Indonesia: Dr. Suryo Anggoro Kusumo Wibowo, Rheumatology Division, Department of Internal Medicine, Fakultas Kedokteran, Universitas Indonesia. Mexico: Dr. Jorge Rojas Serrano, Rheumatologist and clinical investigator, Interstitial Lung Disease and Rheumatology Unit, Instituto Nacional de Enfermedades Respiratorias, Mexico City, Mexico. Dr. Ignacio GarcíaDe La Torre- Departamento de Inmunología y Reumatología, Hospital General de Occidente and University of Guadalajara, Guadalajara, Jalisco, Mexico. Spain: Dr. Erick Adrian Zamora Tehozol; Rheumatology, Medical Care \& Research, Centro Medico Pensiones Hospital, and IMSS, Spain. Dr. Jesús Loarce-Martos, Rheumatology Department, Hospital Universitario Ramón y Cajal, Carretera de Colmenar Viejo, 9, 1 km, 28,043, Madrid, Spain. Dr. Sergio Prieto-González, Department of Internal Medicine, Hospital Clinic of Barcelona, University of Barcelona, Barcelona, Spain. Dr. Albert Gil-Vila, Autoimmune Systemic Unit, Vall d'Hebron General Hospital in Barcelona, Spain. Dr. Raquel Aranega, Autoimmune Systemic Unit, Vall d'Hebron General Hospital in Barcelona, Spain. Japan: Dr. Ran Nakashima, Department of Rheumatology and Clinical Immunology, Graduate School of Medicine, Kyoto University, 54 Shogoin-Kawahara-cho, Sakyo-ku, Kyoto 606-8507, Japan. Electronic address: Dr. Shinji Sato, Division of Rheumatology, Department of Internal Medicine, Tokai University School of Medicine, 143 Shimokasuya, Isehara, 259-1193, Japan. Dr. Naoki Kimura, Department of Lifetime Clinical Immunology, Graduate School of Medical and Dental Sciences, Tokyo Medical and Dental University (TMDU), Tokyo, Japan. Dr. Yuko Kaneko, Division of Rheumatology, Department of Internal Medicine, Keio University School of Medicine, Tokyo, Japan. Germany: Dr. Stylianos Tomaras, Department of Rheumatology, Helios Clinic Vogelsang-Gommern, 39,245 Gommern, Germany. Russian Federation: Dr. Margarita Aleksandrovna Gromova, Pirogov Russian National Research Medical University (RNRMU), Moscow, Russian Federation. Israel: Mr. Or Aharonov, Department of Gerontology, Faculty of Social Welfare and Health Science, University of Haifa, Haifa, Israel.

Author contributions Conceptualisation: LG, VA, RA. Data curation: all authors. Formal analysis: LG. Funding acquisition: not applicable. Investigation: VA, RA, LG, HC, OD, JBL. Methodology: RA, LG, JBL, HC. Software: LG. Validation: VA, RA, JBL, HC. Visualization:
RA, VA, LG. Writing—original draft—PS, LG. Writing—review and editing: all authors.

Funding $\mathrm{HC}$ is supported by the National Institution for Health Research Manchester Biomedical Research Centre Funding Scheme. The views expressed in this publication are those of the authors and not necessarily those of the NHS, the National Institute for Health Research or the Department of Health.

\section{Declarations}

Conflict of interest OD has/had consultancy relationship with and/or has received research funding from or has served as a speaker for the following companies in the area of potential treatments for systemic sclerosis and its complications in the last 3 years: Abbvie, Acceleron, Alcimed, Amgen, AnaMar, Arxx, Baecon, Blade, Bayer, Boehringer Ingelheim, ChemomAb, Corbus, CSL Behring, Galapagos, Glenmark, GSK, Horizon (Curzion), Inventiva, iQvia, Kymera, Lupin, Medac, Medscape, Mitsubishi Tanabe, Novartis, Roche, Roivant, Sanofi, Serodapharm, Topadur and UCB. Patent issued "mir-29 for the treatment of systemic sclerosis" (US8247389, EP2331143). The rest of the authors have no COI relevant to this manuscript.

Ethics approval Ethical approval was obtained from the Institutional Ethics Committee of Sanjay Gandhi Postgraduate Institute of Medical Sciences, Raebareli Road, Lucknow, 226014 on 08-06-2021 (IEC Code: 2021-143-IP-EXP-39).

Disclaimer No part of this manuscript has been copied or published elsewhere in whole or in part.

\section{References}

1. Anderson RM, Vegvari C, Truscott J, Collyer BS (2020) Challenges in creating herd immunity to SARS-CoV-2 infection by mass vaccination. The Lancet 396:1614-1616. https://doi.org/10. 1016/S0140-6736(20)32318-7

2. Velikova T, Georgiev T (2021) SARS-CoV-2 vaccines and autoimmune diseases amidst the COVID-19 crisis. Rheumatol Int 41:509-518. https://doi.org/10.1007/s00296-021-04792-9

3. Gianfrancesco M, Hyrich KL, Al-Adely S et al (2020) Characteristics associated with hospitalisation for COVID-19 in people with rheumatic disease: data from the COVID-19 Global Rheumatology Alliance physician-reported registry. Ann Rheum Dis 79:859-866. https://doi.org/10.1136/annrheumdis-2020-217871

4. Gupta L, Lilleker JB, Agarwal V et al (2021) COVID-19 and myositis - unique challenges for patients. Rheumatol (Oxf) 60:907-910. https://doi.org/10.1093/rheumatology/keaa610

5. Gupta L, Kharbanda R, Agarwal V et al (2021) Patient perspectives on the effect of the SARS-CoV-2 pandemic on patients with systemic sclerosis: an international patient survey. J Clin Rheumatol 27:31-33. https://doi.org/10.1097/RHU.0000000000001681

6. Gaur PS, Gupta L (2020) Changing research paradigm in the face of a global pandemic: foreseeable impact and adaptive measures in academic research in the future. Proc Shevchenko Sci Soc Med Sci. https://doi.org/10.25040/ntsh2020.02.05

7. Gupta L, Gadiwala SF (2021) Coping with the coronavirus disease-2019 pandemic: A giant leap towards digital transformation in academic research. Indian J Rheumatol 16:123. https://doi.org/ 10.4103/injr.injr_251_20

8. ICMR_Ethical_Guidelines_2017.pdf 
9. Eysenbach G (2004) Improving the quality of web surveys: the checklist for reporting results of internet E-surveys (CHERRIES). J Med Internet Res 6:e132. https://doi.org/10.2196/jmir.6.3.e34

10. Gaur PS, Zimba O, Agarwal V, Gupta L (2020) reporting survey based studies - a primer for authors. J Korean Med Sci. https:// doi.org/10.3346/jkms.2020.35.e398

11. Sonani B, Aslam F, Goyal A et al (2021) COVID-19 vaccination in immunocompromised patients. Clin Rheumatol 40:797-798. https://doi.org/10.1007/s10067-020-05547-w

12. Khan H, Gasparyan AY, Gupta L (2021) Lessons learned from publicizing and retracting an erroneous hypothesis on the Mumps, Measles, Rubella (MMR) vaccination with unethical implications.
J Korean Med Sci 36:e126. https://doi.org/10.3346/jkms.2021.36. e126

13. Goel A, Gupta L (2020) Social media in the times of COVID-19. J Clin Rheumatol 26:220-223. https://doi.org/10.1097/RHU.00000 00000001508

14. Tariq J, Gupta L (2021) Safety and efficacy of COVID-19 vaccines in pregnant women with rheumatic diseases: an immunologic perspective. Rheumatol Int 41:1545-1547. https://doi.org/10.1007/ s00296-021-04918-z

Publisher's Note Springer Nature remains neutral with regard to jurisdictional claims in published maps and institutional affiliations.

\section{Authours and Affiliations}

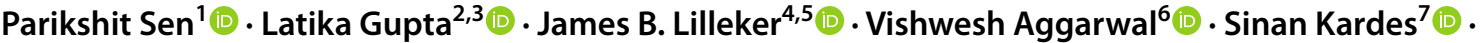

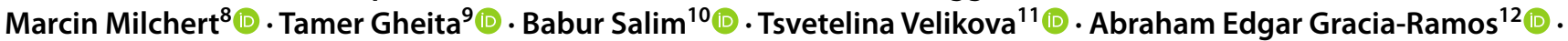

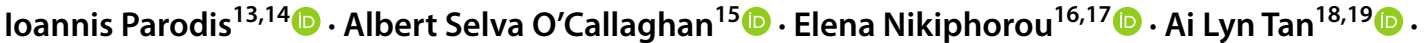

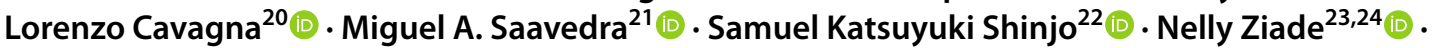

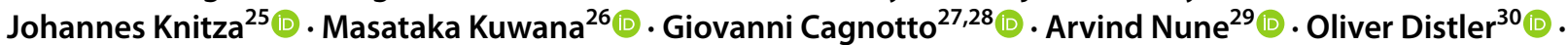

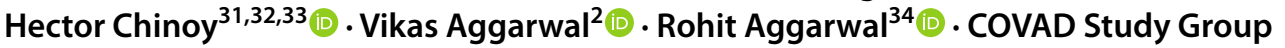

1 Maulana Azad Medical College, 2-Bahadurshah Zafar Marg, New Delhi, Delhi 110002, India

2 Department of Clinical Immunology and Rheumatology, Sanjay Gandhi Postgraduate Institute of Medical Sciences, Rae Bareilley Road, Lucknow 226014, India

3 Department of Rheumatology, Royal Wolverhampton Hospitals NHS Trust, Wolverhampton WV10 0QP, UK

4 Division of Musculoskeletal and Dermatological Sciences, School of Biological Sciences, Faculty of Biology, Medicine and Health, Centre for Musculoskeletal Research, Manchester Academic Health Science Centre, The University of Manchester, Manchester, UK

5 Manchester Centre for Clinical Neurosciences, Salford Royal NHS Foundation Trust, Salford, UK

6 Mahatma Gandhi Mission Medical College, Navi Mumbai, Maharashtra, India

7 Department of Medical Ecology and Hydroclimatology, Istanbul Faculty of Medicine, Istanbul University, Capa-Fatih, Istanbul 34093, Turkey

8 Department of Rheumatology, Internal Medicine, Geriatrics and Clinical Immunology, Pomeranian Medical University, ul Unii Lubelskiej 1, 71-252, Szczecin, Poland

9 Rheumatology Department, Kasr Al Ainy School of Medicine, Cairo University, Cairo, Egypt

10 Rheumatology Department, Fauji Foundation Hospital, Rawalpindi, Pakistan

11 Department of Clinical Immunology, Medical Faculty, University Hospital "Lozenetz", Sofia University St. Kliment Ohridski, 1 Kozyak Str., Sofia 1407, Bulgaria

12 Department of Internal Medicine, General Hospital, National Medical Center "La Raza", Instituto Mexicano del Seguro Social, Av. Jacaranda S/N, Col. La Raza, Del. Azcapotzalco, C.P. 02990 Mexico City, Mexico
13 Division of Rheumatology, Department of Medicine Solna, Karolinska Institutet and Karolinska University Hospital, Stockholm, Sweden

14 Department of Rheumatology, Faculty of Medicine and Health, Örebro University, Örebro, Sweden

15 Internal Medicine Department, Vall D'hebron General Hospital, Universitat Autonoma de Barcelona, 08035 Barcelona, Spain

16 Centre for Rheumatic Diseases, King's College London, London, UK

17 Rheumatology Department, King's College Hospital, London, UK

18 NIHR Leeds Biomedical Research Centre, Leeds Teaching Hospitals Trust, Leeds, UK

19 Leeds Institute of Rheumatic and Musculoskeletal Medicine, University of Leeds, Leeds, UK

20 Rheumatology Unit, Dipartimento di Medicine Interna e Terapia Medica, Università Degli Studi di Pavia, Pavia, Lombardy, Italy

21 Departamento de Reumatología Hospital de Especialidades Dr. Antonio Fraga Mouret, Centro Médico Nacional La Raza, IMSS, Mexico City, Mexico

22 Division of Rheumatology, Faculdade de Medicina FMUSP, Universidade de Sao Paulo, São Paulo, SP, Brazil

23 Rheumatology Department, Saint-Joseph University, Beirut, Lebanon

24 Rheumatology Department, Hôtel-Dieu de France Hospital, Beirut, Lebanon

25 Medizinische Klinik 3 - Rheumatologie und Immunologie, Universitätsklinikum Erlangen, Friedrich-Alexander-Universität Erlangen-Nürnberg, Ulmenweg 18, 91054 Erlangen, Germany 
26 Department of Allergy and Rheumatology, Nippon Medical School Graduate School of Medicine, 1-1-5 Sendagi, Bunkyo-ku, Tokyo 113-8602, Japan

27 Rheumatology, Department of Clinical Sciences, Lund University, Malmö, Sweden

28 Department of Rheumatology, Skåne University Hospital, Lund, Sweden

29 Southport and Ormskirk Hospital NHS Trust, Southport PR8 6PN, UK

30 Department of Rheumatology, University Hospital Zurich, University of Zurich, Zurich, Switzerland

31 Division of Musculoskeletal and Dermatological Sciences, Centre for Musculoskeletal Research, School of Biological Sciences, The University of Manchester, Manchester, UK
32 National Institute for Health Research Manchester Biomedical Research Centre, Manchester University NHS Foundation Trust, The University of Manchester, Manchester, UK

33 Department of Rheumatology, Salford Royal NHS Foundation Trust, Manchester Academic Health Science Centre, Salford, UK

34 Division of Rheumatology and Clinical Immunology, Department of Medicine, UPMC Arthritis and Autoimmunity Center, School of Medicine, University of Pittsburgh, 3601 Fifth Ave., Suite 2B, Pittsburgh, PA 15213, USA 